\title{
Shallow flow over a bed with a lateral change of roughness
}

\author{
Victor Dupuis ${ }^{1, *}$, Frédéric Moulin ${ }^{1, * *}$, Sébastien Cazin ${ }^{1}$, Moïse Marchal $^{1}$, Pierre Elyakime ${ }^{1}$, \\ Jean-Dominique Barron ${ }^{1}$, and Olivier Eiff ${ }^{2, * * *}$ \\ ${ }^{1}$ Institute for Fluid Mechanics (IMFT), University of Toulouse, CNRS, Toulouse, France \\ ${ }^{2}$ Karlsruhe Institute for Technology (KIT), Institute for Hydromechanics, Karlsruhe, Germany
}

\begin{abstract}
River beds frequently exhibit a lateral variation of roughness. For example, in the case of an overflowing river, the main channel has a smoother topography compared to the adjacent floodplains where vegetation and land occupation yield an important hydraulic roughness. The lateral difference in roughness can induce a high lateral velocity gradient within the river crosssection that gives birth to a mixing layer. This mixing layer leads to fluid and momentum transfers between the two adjacent beds. To understand such mixing processes in rivers is important for predicting stage-discharge relationships and the velocity distribution within the cross-section. In order to address these issues in the context of a shallow water flow with a water depth $h$ of the same order as the roughness elements of the bed, experiments were undertaken in a $26 \mathrm{~m}$ long and $1.1 \mathrm{~m}$ wide glass-walled open-channel flume. One half-side of the bed was covered with an array of cubes of height $k$ arranged in a square configuration, the other side with smooth glass. Three different levels of cube submergence $h / k$ were examined $(h / k=0.8,1.5$ and 2$)$. The experiments and measurements were designed to yield the flow in the complete volume of the interstices across the cube array. To achieve this, 2C-3D linear-scanning PIV measurements with zero-parallax optics were developed and set up. The measurements revealed the complexity of the flow structure around the interface between the rough and smooth beds. The results show that the ability of the mixing layer to exchange momentum is highly dependent on the level of the cube submergence $h / k$.
\end{abstract}

\section{Introduction}

Consider a river bed that can be laterally separated into a rough part and a smooth part. To compute the flow in such a bed, one can decompose the section in two adjacent beds separated by a fictive wall, each having a uniform roughness. Yen [1] reports several formula to compute the discharge in such a way. But this approach does not take into account the dynamical interaction between the two adjacent beds. In fact this interaction can be very important due to the generation of a high velocity gradient between the two beds, which

\footnotetext{
*e-mail: victor.dupuis@imft.fr

**e-mail: frederic.moulin@imft.fr

***e-mail: olivier.eiff@kit.edu
} 
can cause a mixing layer to develop at the interface. The effect of the mixing layer is to redistribute the velocity in the cross-section, bringing in high-velocity fluid into the rougher part of the bed and conversely reducing momentum in the smooth part of the bed. To be able to predict the correct velocity distribution in the river cross-section is important for example for studying fish habitat, for computing the scour of an erodible bed, or evaluating the load on structures during floods.

Vermaas et al. [2] studied the development of a mixing layer between a smooth bed and a bed roughened by a layer of stones. White and Nepf [3] did the same for a smooth bed adjacent to an array of emergent cylinders, where the solid volume fraction varied from $N=$ 0.02 to 0.10 . Both studies reported high momentum exchange between the two beds and the presence of large-scale turbulent structures. Vermaas et al. [2] also stressed that, additionally to the mixing layer, secondary currents can also play a major role in redistributing momentum within the cross-section.

The objective of the present study is to investigate the momentum exchange between a smooth bed and a bed roughened with macro-roughness elements and a high solid volume fraction $(N=0.19)$. Both cases with immerged and emergent roughness elements are considered. The cubic macro-roughness elements aim at representing macro-obstacles present in the river bed or floodplain, like buildings or dense bushes in the case of floods. The scientific issue is to describe and explain how the momentum is redistributed by the lateral exchange processes in such river-like flows.

Flows through and above roughness elements are characterized by highly variable space and time scales. To analyse such flows, double-averaging, i.e. time averaging (classical Reynolds-averaging) combined with spatial averaging, is often required (Nikora et al. [4]). Time-averaging is easy to carry out with classical measuring tools (ADV, LDV, PIV). Spatial averaging in a plane can also be performed easily with a PIV technique, provided the length scale over which the spactial averaging has to be performed is smaller than the measured velocity field. In turn, volumetric averaging is much more difficult to implement. Tomographic 3D-3C PIV (three dimensions, three components) is an appropriate technique to perform volumetric averaging, but it requires a complex apparatus and the typical size of the measuring volume can hardly exceed $10 \mathrm{~cm}$ (e.g. Schröder et al. [5], Gao et al. [6]). Single-point measurement systems like ADV or LDV can also be used for spatial averaging, measuring over a sufficiently refined mesh, but the measurement time often becomes excessively long. Another way to perform volumetric averaging is PIV scanning, which has been used for several years with different techniques (Brücker [7], Liberzon et al. [8], Albagnac et al. [9]). In this paper we present a derivative of the technique used by Albagnac et al. [9], a new spatially 3D-2C PIV scanning technique, which is relatively simple to set up and yields accurate and reliable results. In addition to a classical 2D-2C PIV system, this technique requires a linear motor with accurate positioning and telecentric lenses for the camera and laser sheet.

\section{Experimental setup}

The experiments are performed in a $26 \mathrm{~m}$ long and $1.1 \mathrm{~m}$ wide glass-walled open-channel flume at IMFT, Toulouse. The flume has a constant slope of $3.1 \mathrm{~mm} \cdot \mathrm{m}^{-1}$. As sketched in Fig. 1, one half-side of the bed is smooth glass and the other side is smooth glass covered with an array of cubes of side $k=40 \mathrm{~mm}$ arranged in a square configuration. The elementary pattern of this arrangement in the horizontal plane is a $91.5 \times 91.5 \mathrm{~mm}^{2}$ square, yielding a solid volume fraction of $N=0.19$. We note $x$ the longitudinal direction parallel to the bed, $y$ the transverse direction with $y=0$ at the interface between the array and the smooth bed (the width of the array being 6 entire lengths of the elementary pattern) and $z$ the normal to the bed, with $z=0$ at the glass bed level. The cubes in front of the camera field of view are 
transparent in order to visualize the flow between the cubes. The other cubes are made of PVC.

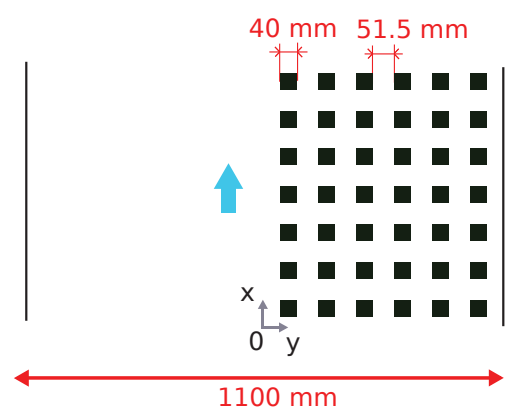

Figure 1. Top view of the channel bed configuration.

Velocities in the longitudinal $(u)$ and vertical $(w)$ directions are measured using a new 3D2C scanning Particle Image Velocimetry (PIV) technique. The measurement setup, placed at $x=19.5 \mathrm{~m}$ from the channel inlet, is sketched in Fig. 2. A continuous laser, combined with a cylindrical divergent lens and a telecentric lens, generates a collimated laser sheet of 2.7 $\mathrm{mm}$ thickness and $30 \mathrm{~cm}$ width, the collimation avoiding shadows at the cubes' edges. The laser sheet enters from the bottom of the channel after being reflected by a $45^{\circ}$-positionedmirror. This $45^{\circ}$-mirror is mounted on a linear motor that can travel with high speed and high positioning accuracy in the $y$-direction. The laser sheet in the $x z$-plane is viewed by a camera placed at the side of the flume. A second telecentric lens mounted on the camera prevents parallax effects on the image, i.e. it allows looking through the cubes without shadows. Moreover, the image calibration (pixel-to-millimetre conversion) is the same for different $y$ positions of the mirror. The water is seeded with $60 \mu \mathrm{m}$ diameter polyamide particles of density 1.03 .

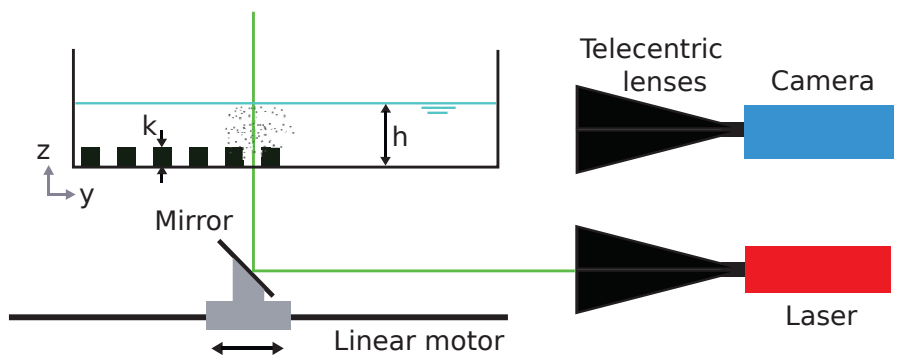

Figure 2. 3D-2C scanning PIV setup.

This arrangement enables a three-dimensional two-component (3D-2C) velocity measurement. The frame rate of the camera and the travelling velocity of the linear motor are chosen such that the positions of the laser sheet of two consecutive frames overlap to a given percentage. The PIV correlation is then computed between these two successive frames. The lower the overlap between two consecutive frames, the lower the measurement accuracy, since fewer particles are present in both frames. On the other hand, a high overlap involves an increased number of images for the same scanning length, which causes excessive data storage. Thus a compromise was made and an overlap of $85 \%$ was chosen. Figure 3 shows 
the rate of false vectors with increasing overlap. For $85 \%$ overlap, the false vector rate is comparable to classic 2D-2C PIV (100\% laser sheet overlap).

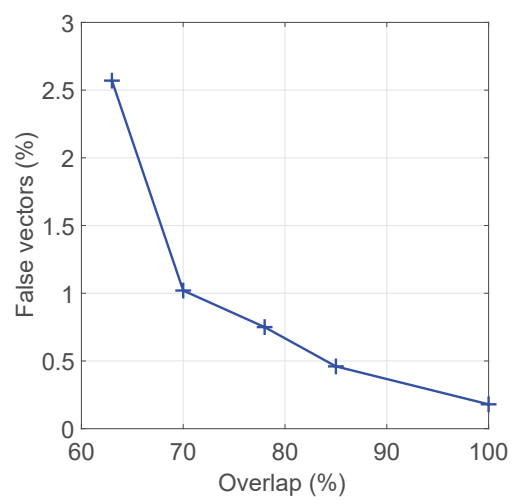

Figure 3. Rate of false vectors as a function of overlap.

If we note $e$ the laser-sheet thickness and $d y$ the displacement of the laser sheet between two frames, then the overlap $B$ is defined by $B=(e-d y) / e$. The frame rate $f$ of the camera is determined by $f=U_{f} / d l$, where $U_{f}$ is the typical flow velocity and $d l$ is the displacement of a particle between two frames, corresponding to a chosen pixel displacement (e.g. 15 pixels). In the time delay $d t=1 / f$ between two frames, the laser sheet must travel a distance $d y$, thus the motor velocity is given by $U_{m o t}=d y / d t=f e(1-B)$.

The camera is triggered by the motor. In practice, the motor is first accelerated along a ramp before reaching the constant velocity $U_{m o t}$. As soon as the motor velocity reaches the plateau $U_{m o t}$ (at known $y$-position), the motor sends a signal to the camera to begin the frame acquisition. Conversely, the motor triggers the recording end as soon as it reaches the end of the scanning length.

Because the depth of focus of the camera telecentric lens is limited to $200 \mathrm{~mm}$, the $1100 \mathrm{~mm}$ wide cross-section was divided in four parts, in each of which a scanning measurement was made. These four measuring volumes were then merged together to get a quasi complete cross-section. For technical reasons the region $y>410 \mathrm{~mm}$ could not be measured; moreover, because of reflections of the laser sheet on the free-surface, the last top $15 \mathrm{~mm}$ near the free-surface could not be measured as well. The measuring volume is about $200 \mathrm{~mm}$ large in the $x$-direction.

Three test cases were investigated, with different cube submergences: two submerged cases with $h / k=2$ and 1.5 (test cases SUB2 and SUB1), where $h$ is water depth; and an emergent case with $h / k=0.8$ (test case EMG). Table 1 summarizes the flow conditions for the three test cases: total discharge $Q_{t o t}$, subsection bulk velocity $U_{Q, i}(i=a$ for the cube array and $i=s$ for the smooth part), as well as Froude and Reynolds numbers in each subsection.

For each test case, about 110 measuring volumes were recorded (one volume contains about 2400 PIV fields with a lateral spacing of $d y=0.4 \mathrm{~mm}$ ). Each volume can be considered as a statistically independent sample since the time between two scans exceeds the highest time scale of the flow. These 110 samples were sufficient to obtain relatively good converged values of mean velocities and Reynolds stress tensor components.

The PIV images were processed with the software CPIV-IMFT developed at IMFT. CPIVIMFT is a parallelised PIV correlation algorithm using fast Fourier transforms. 
Table 1. Flow conditions of the test cases.

\begin{tabular}{cccccccccc}
\hline Test case & $h / k$ & $\begin{array}{c}h \\
(\mathrm{~mm})\end{array}$ & $\begin{array}{c}Q_{\text {tot }} \\
\left(\mathrm{L} . \mathrm{s}^{-1}\right)\end{array}$ & $\begin{array}{c}U_{Q, a} \\
\left(\mathrm{~cm} . \mathrm{s}^{-1}\right)\end{array}$ & $\begin{array}{c}U_{Q, s} \\
\left(\mathrm{~cm} . \mathrm{s}^{-1}\right)\end{array}$ & $F r_{a}$ & $F r_{s}$ & $R e_{a}$ & $R e_{s}$ \\
\hline SUB2 & 2 & 80 & 45.5 & 23.7 & 78.9 & 0.28 & 0.89 & $78.5 \times 10^{3}$ & $252.4 \times 10^{3}$ \\
SUB1 & 1.5 & 60 & 26.2 & 16.8 & 66.1 & 0.17 & 0.86 & $32.0 \times 10^{3}$ & $158.5 \times 10^{3}$ \\
EMG & 0.8 & 32 & 10.5 & 11.4 & 52.8 & 0.12 & 0.94 & $8.7 \times 10^{3}$ & $67.6 \times 10^{3}$ \\
\hline
\end{tabular}

\section{Cross-sectional velocity distribution}

Owing to the scanning technique a complete overview of the flow organization in the channel cross-section can be obtained. Figure 4 shows the distribution of longitudinal velocity and longitudinal turbulence intensity in the cross-section for the three test cases. Both quantities are spatially averaged in the longitudinal direction along the length of one cube array pattern $(91.5 \mathrm{~mm})$. The spatial average is intrinsic, i.e. over the volume of fluid (Nikora $e t$ al. [4]). The investigated flows are characterized by strong turbulence production at the interface between the smooth bed and the cube array, as seen on Fig. 4 (right). Furthermore, time-resolved PIV measurements (not presented here) reveal coherent large-scale velocity fluctuations at the interface, that are typical of mixing layers in shallow flows (White and Nepf [3], Dupuis et al. [11]). This indicates that for each test case a mixing layer develops at the interface, generated by the high difference in velocity between the two flow regions.
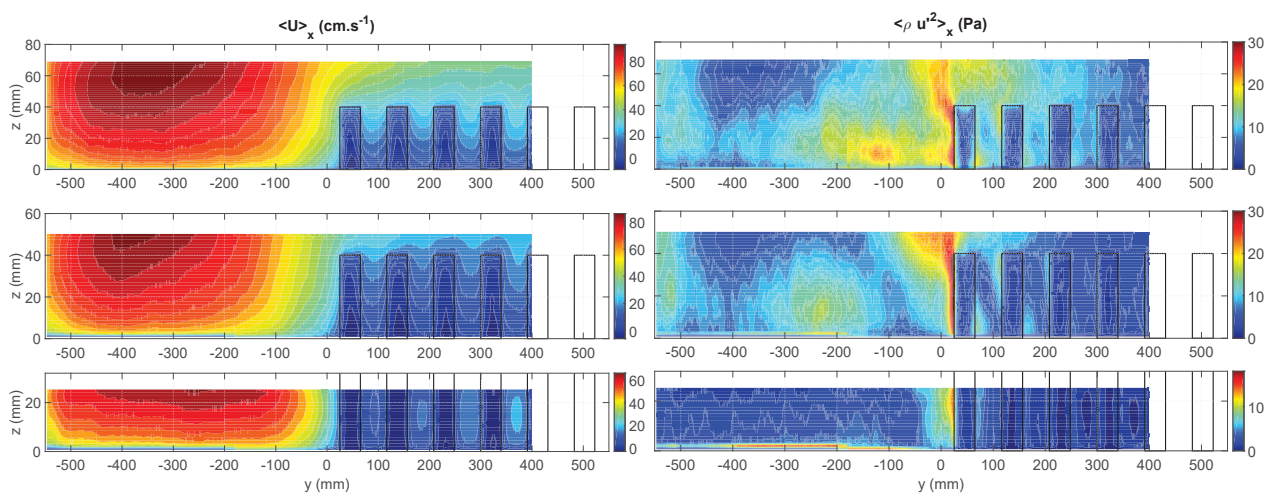

Figure 4. Cross-section distribution of mean longitudinal velocity (left) and longitudinal turbulence intensity (right) for test cases SUB2 (top), SUB1 (middle) and EMG (bottom). The two quantities are spatially averaged in longitudinal direction. Scale is distorted.

By integrating the mean longitudinal velocity in the cross-section (after extrapolating the domain without measurement corresponding to the white zones in Fig. 4) the discharge in each subsection can be obtained: $Q_{s}$ in the smooth part $(y<0)$ and $Q_{a}$ in the part with the array $(y>0)$. The partial discharges $Q_{s}$ and $Q_{a}$ are reported in Table 2. Not surprisingly, the ratio $Q_{a} / Q_{t o t}$ decreases with decreasing cube submergence. For the emerged test case EMG, the discharge in the cube array is only $6.8 \%$ of the total discharge although the array occupies the half width of the channel.

Consider the discharge $Q_{2 D, i}$ that would be obtained in each subsection $i=s, f$ if a wall would separate at $y=0$ the cross-section into two non-interacting flows, the water depth being the same. This discharge is computed with a Manning formula for the smooth part, 
Table 2. Subsection discharge distribution and comparison with a fictive wall flow.

\begin{tabular}{cccccccc}
\hline Test case & $\begin{array}{c}Q_{a} \\
\left(\mathrm{~L} . s^{-1}\right)\end{array}$ & $\begin{array}{c}Q_{s} \\
\left(\mathrm{~L} . \mathrm{s}^{-1}\right)\end{array}$ & $\begin{array}{c}Q_{a} / Q_{\text {tot }} \\
(\%)\end{array}$ & $\begin{array}{c}Q_{2 D, a} \\
\left(\mathrm{~L} . \mathrm{s}^{-1}\right)\end{array}$ & $\begin{array}{c}Q_{2 D, s} \\
\left(\mathrm{~L} . \mathrm{s}^{-1}\right)\end{array}$ & $\begin{array}{c}\frac{Q_{a}-Q_{2 D, a}}{Q_{2 D, a}} \\
(\%)\end{array}$ & $\begin{array}{c}\frac{Q_{s}-Q_{2 D, s}}{Q_{2 D, s}} \\
(\%)\end{array}$ \\
\hline SUB2 & 10.8 & 34.7 & 24.6 & 9.0 & 40.0 & 20.0 & -13.3 \\
SUB1 & 4.4 & 21.8 & 13.3 & 4.5 & 25.7 & -2.2 & -15.2 \\
EMG & 1.2 & 9.3 & 6.8 & 1.2 & 9.6 & 0.0 & -3.1 \\
\hline
\end{tabular}

using a Manning coefficient of $n=0.0096 \mathrm{~s} . \mathrm{m}^{-1 / 3}$ (smooth glass bed). For the cube array, we use the measurements of Chagot et al. [10] who investigated the same cube array occupying the whole flume width. Table 2 reports the relative difference in discharge for each subsection relative to the flow with a fictive wall $\left(Q_{i}-Q_{2 D, i}\right) / Q_{2 D, i}$. For test case SUB2, the discharge in the smooth part is decreased by the interaction (by $13 \%$ ) and the discharge in the rough part is increased (by $20 \%$ ). This behaviour can be explained by the mixing layer at the interface between the two beds which transfers low-speed fluid from the cube array to the smooth bed, and reversely high-speed fluid from the smooth bed to the cube array. For test cases SUB1 and EMG, no increase of the discharge is detected in the cube array, although a mixing layer is present as well. The absence of an accelerating effect by the mixing layer in the cube array for test cases SUB1 and EMG will be analysed in the next section.

\section{Lateral profiles of flow quantities}

The data obtained by the scanning PIV technique also enables to plot transverse profiles of double-averaged flow quantities at fixed elevations $z$. Figure 5 shows the transverse profiles of the mean longitudinal velocity (left) and longitudinal turbulence intensity (right) at zelevations above and below the cube top, for the three test cases. The quantities are spatially averaged either in the $x$-direction and noted $\langle\cdot\rangle_{x}$ (blue and red lines) or in both the $x$ - and $y$-directions and noted $\langle\cdot\rangle_{x, y}$ (green and black lines). This latter averaging allows to smooth out the spatial variations due to the cube and to examine the spatial variations due to the horizontal mixing layer only. Below the cube top, the cubes' wakes are clearly visible and characterised by a strong dip in the $\langle U\rangle_{x}$-velocity and by increased longitudinal turbulence along the side faces of the cubes. Sufficiently above the cube, the spatial variations related to the presence of the cubes are significantly reduced, i.e. the flow is above the roughness sublayer.

Above the cubes (for test cases SUB1 and SUB2), the lateral profile of velocity in the region $-300<y<300 \mathrm{~mm}$ has a typical shape for a mixing layer: a monotonous decrease from the high-speed-side to the low-speed-side with an inflection point. The profile is asymmetric with respect to the inflection point, which is typical for shallow mixing layers (Dupuis et al. [11]). Below the cubes' top, the profile of $\langle U\rangle_{x, y}$ in the zone $150<y<300 \mathrm{~mm}$ depends on submergence $h / k$. In this zone $\langle U\rangle_{x, y}$ is constant for SUB2, slightly increasing towards the wall for SUB1 and noticeably increasing for EMG. This can be explained as follows. For cubes away from the interface, the flow in the free corridor (between two rows of cubes) is mainly unidirectional in the $x$-direction. Near the interface however, the flow is more turbulent, as shown in Fig. 5 (right), and is characterised by strong and long-lived sweeps and ejections (as revealed by the time-resolved PIV measurements). These sweeps and ejections, which are typical for mixing layers (White and Nepf [3]), induce high-intensity lateral cross-flows in the main corridors, such that a unidirectional flow cannot develop and the mean velocity is reduced in the free corridor. The velocity in the cube array is therefore 

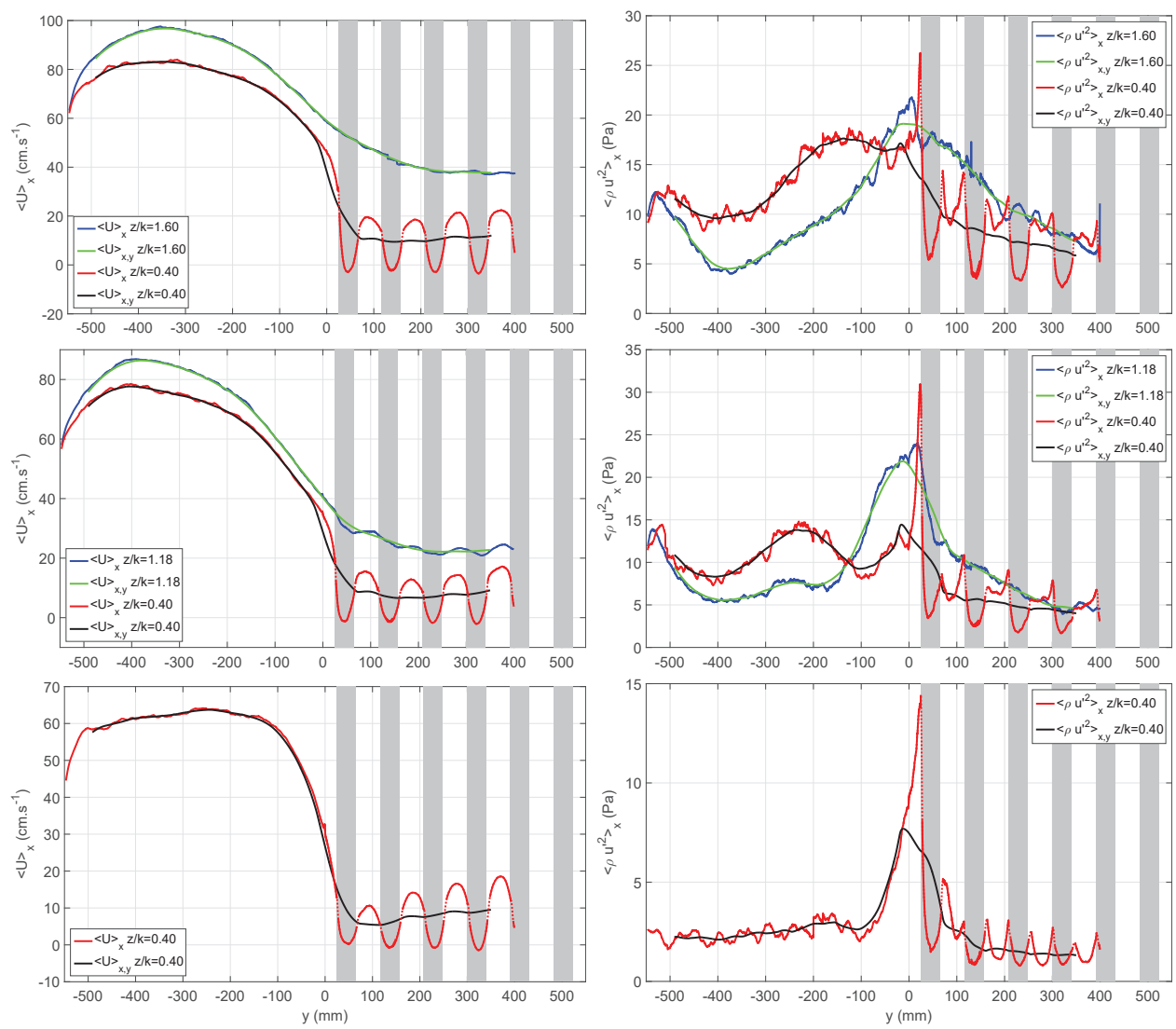

Figure 5. : Transverse profiles of mean longitudinal velocity (left) and longitudinal turbulence intensity (right) at fixed $z$-elevations above and below the cube top $k$. Test case SUB2 (top), SUB1 (middle) and EMG (bottom). For test case EMG, there is no flow above the cubes. The quantities are either only longitudinally averaged $\left(\langle\cdot\rangle_{x}\right)$ or both longitudinally and laterally averaged $\left(\langle\cdot\rangle_{x, y}\right)$. Grey bands indicate the position of the cubes.

driven by two opposite effects of the mixing layer: the acceleration due to inflow of highspeed fluid and the deceleration due to the perturbation of the flow in the free corridor. Very close to the interface $(0<y<100 \mathrm{~mm})$, the first effect is dominant, such that $\langle U\rangle_{x, y}$ increases when approaching the interface for all test cases. In the zone $150<y<300 \mathrm{~mm}$, the second effect becomes dominant for test cases SUB1 and EMG, whereas for test case SUB2 velocity $\langle U\rangle_{x, y}$ is constant, indicating that both effects balance each other out.

For test case EMG, the combination of these two effects is clearly visible when analysing the $\langle U\rangle_{x}$-velocity: for cubes close to the interface, the velocity dip in the cubes' wake is less pronounced than for outer cubes (inflow of high-speed fluid) but the velocity peak in the main corridor is lower (due to cross-flows). For test cases SUB2 and SUB1, the velocity dip and velocity peak are almost the same for all cubes, indicating that the horizontal mixing layer has little influence on the flow below the cube top. This may be due to a third effect which is the forcing due to the high-speed flow above the cubes, that could help to sustain a unidirectional flow in the free corridors even close to the interface. 


\section{Conclusion}

Flows in a river bed featuring a lateral smooth-to-rough change were experimentally studied in a $26 \mathrm{~m}$ long flume. One half of the bed was covered with a cube array representing macro-roughness elements like bushes or buildings on the floodplains. The other bed half was smooth, representing the main channel. Three flow conditions with both low submerged and emergent roughness elements were considered.

A 3D-2C scanning PIV technique was developed for investigating these complex flows. This technique allows to measure two velocity components in a large measurement volume, even in the presence of obstacles in the flow. In addition to a classic 2D-2C PIV system, a linear motor and zero-parallax optics for the camera and laser sheet are needed for achieving the setup.

Owing to the scanning technique, a complete overview of the double-averaged velocity and turbulence intensity in the flume cross-section was obtained. The mixing layer that develops at the interface between the cube array and the smooth bed induces (1) an important turbulence level at the interface, and (2) an exchange of mean momentum between the flows in the two half-beds. However, the effect of the mixing layer in accelerating the flow in the rough bed strongly depends on the cubes' submergence. For a submergence of $h / k=2$, the mixing layer accelerates the flow on the rough side, relative to what is obtained by modelling the flow without a mixing layer. For lower submergence $(h / k=1.5)$ and for the emergent case, no mixing-layer induced acceleration of the flow on the rough side is observed. The proposed explanation is that the accelerating effect of the mixing layer (inflow of high-speed fluid from the smooth bed) is balanced by the perturbation of the flow in the inter-cube region (main corridor), where strong cross-flows due to sweep and ejection events prevent a unidirectional flow.

\section{Acknowledgements}

This work was funded by the European Union (Hydralab+ project) and by the French National Research Agency (Flowres project, grant No. ANR-14-CE03-0010). The data were processed at the academic supercomputing centre CALMIP.

\section{References}

[1] B.C. Yen, Journal of Hydraulic Engineering 128, 20 (2002)

[2] D. Vermaas, W. Uijttewaal, A. Hoitink, Water Resources Research 47 (2011)

[3] B.L. White, H.M. Nepf, Journal of Fluid Mechanics 593, 1 (2007)

[4] V. Nikora, I. McEwan, S. McLean, S. Coleman, D. Pokrajac, R. Walters, Journal of Hydraulic Engineering 133, 873 (2007)

[5] A. Schröder, R. Geisler, K. Staack, G. Elsinga, F. Scarano, B. Wieneke, A. Henning, C. Poelma, J. Westerweel, Experiments in Fluids 50, 1071 (2011)

[6] Q. Gao, C. Ortiz-Dueñas, E.K. Longmire, Experiments in Fluids 54, 1625 (2013)

[7] C. Brücker, Applied Scientific Research 56, 157 (1996)

[8] A. Liberzon, R. Gurka, G. Hetsroni, Experiments in Fluids 36, 355 (2004)

[9] J. Albagnac, F.Y. Moulin, O. Eiff, L. Lacaze, P. Brancher, Environmental Fluid Mechanics 14, 957 (2014)

[10] L. Chagot, F. Moulin, O. Eiff, 4th Int. Symposium of Shallow Flows, Eindhoven (2017)

[11] V. Dupuis, S. Proust, C. Berni, A. Paquier, Experiments in Fluids 58, 30 (2017) 